\title{
Gestión del conocimiento de social media marketing, para aumentar las ventas de las micro-empresas de servicios, cuyos clientes son parte de una comunidad universitaria. Caso Ciudad de Puebla.
}

\section{Management of social media marketing, to increase sales in service SMEs, whose clients are part of a university community. Puebla City case study.}

Karla-Coré Moreno-Cortés ${ }^{1 ;}$; María-Martha del Socorro Romano-Cadena²;

María del Refugio García-Alarcón ${ }^{3}$

Palabras clave:

Social media marketing, ventas de servicios, comunidad universitaria, microempresas.

Artículo de investigación:

Fecha de recepción: 2019/10/13

Fecha de aceptación: 2019/12/16

Esta publicación se encuentra bajo licencia:

Creative Commons ReconocimientoNoComercialSinObraDerivada 4.0 Internacional

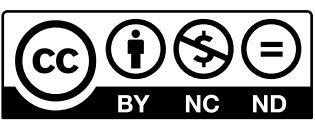

\section{Resumen.}

Las redes sociales ofrecen importantes beneficios en materia de marketing, entre los más importantes está la facilidad para segmentar a detalle los mercados y los bajos costos.

A pesar de ello, las microempresas, las cuales representan un alto índice en México, no cuentan con los conocimientos para explotar en su totalidad las bondades del social media marketing.

El objetivo del artículo es proponer estrategias de social media marketing, para aumentar las ventas en las microempresas de servicios que tienen como clientes potenciales a comunidades universitarias.

Profesora investigadora de la Benemérita Universidad Autónoma de Puebla, México. Twitter: @KarlaCoreM, karla.morenoco@correo.buap.mx, código ORCID: https://orcid.org/0000-0001-5673-2428

2 Profesora investigadora de la Benemérita Universidad Autónoma de Puebla, México. Twitter: @KarlaCoreM, martha.romano@correo.buap.mx, código ORCID: https://orcid.org/0000-0002-2402-0933

3 Profesora investigadora de la Benemérita Universidad Autónoma de Puebla, México. Twitter: @KarlaCoreM, smary_2003buap@hotmail.com, código ORCID: https://orcid.org/0000-0002-2184-3838 


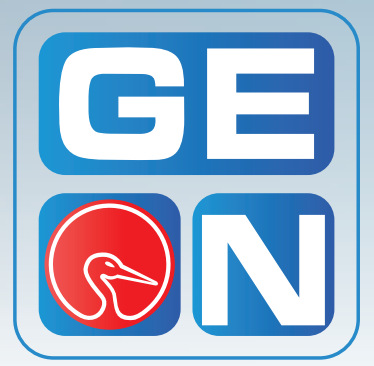

Revista GEON

(Gestión, Organizaciones y Negocios)

ISSN: 2346-3910 en línea

revistageon@unillanos.edu.co

Universidad de los Llanos

Colombia

Moreno Cortés, K., Romano Cadena, M. M., \& García Alarcón, M. (2020).

Gestión del conocimiento de social media marketing, para aumentar las ventas de las micro-empresas de servicios, cuyos clientes son parte de una comunidad universitaria. Caso Ciudad de Puebla

\section{Revista GEON}

(Gestión, Organizaciones y Negocios), 7(1), 112-128.

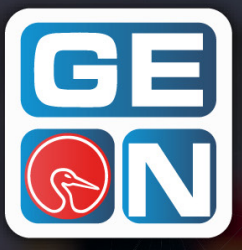

66 las microempresas, las cuales Revista GEON

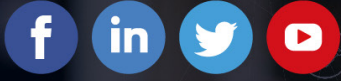
representan un alto índice en México, no cuentan con los conocimientos para explotar en su totalidad las bondades del social media marketing 99

Moreno Cortés, K., Romano Cadena, M. M., \& García Alarcón, M. (2020). Gestión del conocimiento de social media marketing, para aumentar las ventas de las micro-empresas de servicios, cuyos clientes son parte de una comunidad universitaria. Caso Ciudad de Puebla. Revista GEON (Gestión, Organizaciones Y Negocios), 7(1).

\section{http://revistageon.unillanos.edu.co}


La presente investigación utilizó un diseño descriptivo correlacional simple, se hicieron entrevistas a dueños de microempresas y un cuestionario de 7 preguntas aplicado a la comunidad universitaria (clientes potenciales)

Entre los principales resultados, se encontraron que la comunidad universitaria utiliza poco las redes sociales con objetivos de compra, y en los casos en los que han comprado, han sido productos tangibles y no servicios.

Por ello, en las conclusiones del artículo, se presentan estrategias específicas que sean aplicables a las microempresas de servicios y que a su vez puedan ser traducidas en un aumento de las ventas.

Palabras clave: Social media marketing, ventas de servicios, comunidad universitaria, microempresas.

\section{Summary}

Social networks offer important benefits marketing terms, among the most important is the detailed markets segmentation and low publicity costs.

Despite this, SMEs, which represent a high index in Mexico, do not have the knowledge to fully exploit the benefits of social media marketing.

The objective of the article was to propose effective strategies for the implementation of social media marketing, to increase sales in service SMEs that have as potential clients, university communities.

Cómo citar este artículo / Toreference this article:

Moreno Cortés, K., Romano Cadena, M. M., \& García Alarcón, M. (2020). Gestión del conocimiento de social media marketing, para aumentar las ventas de las micro-empresas de servicios, cuyos clientes son parte de una comunidad universitaria. Caso Ciudad de Puebla. Revista GEON (Gestión, Organizaciones Y Negocios), 7(1). 112-128. https://doi. org/10.22579/23463910.186
The present investigation used a simple descriptive correlational design, interviews were conducted with micro-business owners and a 7-question quizz applied to the university community (potential clients)

Among the main results, it was found that the university community uses social networks little with purchase objectives, and in the cases in which they have bought, they have been tangible products but not services. 
Therefore, in the article conclussions, specific strategies applicable to service SMEs are presented, which in turn can be translated into increased sales.

Keywords: Social media marketing, service sales, university community, service SMEs

Códigos JEL: M Business Administration and Business Economics; Marketing; Accounting; Personnel Economics

\section{Introducción.}

Las redes sociales son herramientas estratégicas en el área del marketing de toda empresa, permiten anunciarse de manera masiva, ya que estas redes abarcan millones de usuarios a nivel global, lo cual significa una publicidad masiva a bajo costo.

"La expansión de las tecnologías conllevó el asentamiento en paralelo de una ideología industrial, que aumentó la producción y llegó a sobrepasar su demanda espontánea, con lo que ocasionó una importante inversión de valores. La venta, más que la producción, pasó a ser el objetivo primordial" (Ruiz y Buira, 2007, p.39-47).

De acuerdo al diccionario Zorraquino, el Social Media Marketing consiste en todas aquellas acciones que lleva a cabo una empresa, institución o profesional independiente, para promover sus productos y/o servicios y construir un vínculo con su público objetivo a través de las redes sociales (2019).

El Social Media Marketing (SMM), también conocido como Marketing de
Redes Sociales, es una herramienta estratégica del marketing On-line, asimismo la empresa tiene la posibilidad de establecer una comunicación global e interactiva con sus clientes. Generalmente "se refiere al proceso de ganar tráfico o atención a través del sitios de Social Media. Social Media es un término usado ampliamente para mencionar los sitios que proveen acciones sociales radicalmente diferentes a como se realizaban a inicios de Internet" (Velazquez, 2015)

El Social Media Marketing sirve para generar leads estableciendo contacto a base de llamar la atención en redes sociales.

Su capacidad para atraer grandes masas de usuarios es muy elevada, ya que la viralidad es algo que siempre sobrevuela el campo de las redes sociales. Por tanto, estas estrategias pueden servir para obtener un alto grado de conversiones con suma facilidad (NeoAttack, 2019).

El término lead es un concepto anglosajón que hace referencia al registro de datos de contacto de personas 
Propuesta de una herramienta de emprendimiento

en el diseña de un madela de negacia, IIZ-128
Karla Caré Marena, María Martha del

Socorro Romana, María del Refugio García que están interesadas en nuestra marca o en contenido relacionado con la misma (Díaz, 2019).

El SMM permite vender, conectar con los consumidores, llegar al público correcto, crear contenidos originales, focalizados y de calidad, así como adaptarse a las nuevas tendencias

(Marketing Actual.es, 2015) Los beneficios directos que pueden encontrar las marcas a través del social media marketing son: construir la imagen de marca, maximizar la integración en los medios sociales, conducir tráfico a los sitios web corporativos, tiendas de ecommerce, microsites, desarrollar ideas para nuevas estrategias de negocio, obtener impacto mediático y publicity de forma indirecta, reforzar los vínculos afectivos mediante una relación marca-persona, incrementar el recuerdo y conciencia de marca, mejorar el posicionamiento web mediante links orgánicos, internacionalizar, expandir y abrir nuevos mercados para la marca, incrementar la venta de productos, cumplir objetivos de marketing con mayor eficacia e involucrar a los consumidores en la experiencia de marca.

Todos los beneficios antes mencionados, son especialmente útiles para las microempresas por sus características, tienen poco capital para invertir en marketing y un alto porcentaje de ellas cierran durante sus primeros 5 años de vida.

Las microempresas son todos aquellos negocios que tienen 15 o menos trabajadores, generan anualmente ventas hasta por 4 millones de pesos. En México, representan el 46 por ciento del total de las empresas y el 40 por ciento del empleo en el país; además, producen el 15 por ciento del Producto Interno Bruto (SEECO, 2018).

\section{Problemática encontrada.}

A pesar de que se iniciaron desde 1997, las redes sociales empezaron su popularidad a partir de 2003, en la actualidad, las redes sociales son una parte integral de la vida cotidiana, de acuerdo con (Méndez, F y Mendoza, D, 2018), en México, hay una población de más de 120 millones de habitantes, de los cuales, alrededor de 63 millones están conectados a internet y son usuarios de perfiles en alguna red social

Por tanto, las diferentes plataformas de redes sociales se han convertido en una parte importante de las estrategias de marketing de las empresas como nuevos elementos híbridos de la mezcla de promoción.

El uso de redes sociales para aumentar las ventas, ofrece beneficios claros a las empresas, entre ellos: es más económica que otras formas de marketing; es sencillo medir el impacto de las ventas, en comparación con la inversión; permite flexibilidad de contenidos y uso de fotografías, videos, entre otros medios digitales; generan notoriedad a corto plazo y se pueden crear campañas específicas, es decir ayudan a generar una segmentación estratégica del mercado, basada en datos demográficos y sociodemográficos, intereses, comportamientos, experiencia empresarial, entre otros.

A pesar de lo anteriormente mencionado, existen empresas que aún no 
disfrutan de los beneficios del social media marketing, lo anterior debido a que esta estrategia es relativamente reciente, faltan conocimientos especializados para aplicarla y hay poca confianza de los clientes respecto a comprar a través de redes sociales.

\section{Objetivo.}

Identificar las estrategias de implementación del social media marketing, que conllevan a mejorar el servicio y aumentar las ventas en las microempresas de servicios a través de la investigación de las preferencias de clientes potenciales, pertenecientes a comunidades universitarias.

\section{Contexto teórico.}

A lo largo de la historia los seres humanos se han comunicado de diversas formas desde el lenguaje de señas, hasta la comunicación vía herramientas tecnológicas avanzadas, que permiten presentar e intercambiar información a través de la internet. El internet tiene su origen en septiembre de 1969 se instala en la
Universidad de California (UCLA) el primer procesador de mensajes de interfaz nombrado ARPANET a finales de este mismo año esta crece geométricamente.

Grandes avances tecnológicos se dan durante las décadas de los 70 y 80, en donde aparecen nuevos soportes de almacenamiento, que permiten a los usuarios principalmente instituciones, organizaciones, medianas o pequeñas empresas, construir sus bases de datos.

Las redes sociales que se conforman desde las Tecnologías de Información y Comunicación (TIC), pueden definirse como una estructura social que, apoyada con los recursos de la web, permite las relaciones entre personas, grupos y organizaciones bajo uno o varios objetivos en común; establecimiento de contacto con personas conocidas y con nuevas personas, apoyo emocional, compañía social, ayuda material y de servicio (Peña, Pérez, \& Rondón, 2010). A continuación, se presenta de manera esquemática la cronología de las redes sociales.

Tabla 1. Desarrollo histórico de las redes sociales.

Año Característica

1971 Por primera vez se envía un mail (los dos ordenadores están uno al lado del otro).

1978 A través de la línea telefónica se intercambian mensajes, utilizando el software conocido como Sistema de Tablón de anuncios (Bulletin Board System -BBS). Las copias de navegadores se intercambian a través de la plataforma Usenet

1980 Compu Serve compañía norteamericana la cual fue el primer proveedor de servicios telemáticos, domino el mercado en ésta década.

1990 Aparición de redes mundiales como Fidonet, el BBS más popular hasta la llegada de la Internet

1994 Se crea GeoClties una de las primeras redes sociales, el cual es un sistema clasificador de webs geolocalizado. 
Tabla 1. Desarrollo histórico de las redes sociales. (...Continuación)

\begin{tabular}{|c|c|}
\hline Año & Característica \\
\hline 995 & $\begin{array}{l}\text { Aumentan los BBS gracias al sistema operativo multitareas, v.gr. AmigaOS, OS/2, Windows 3.0, } \\
\text { Unix, Windows 95, 3.1, etc., The Globe.com ofrece a sus usuarios personalizar sus propios con- } \\
\text { tenidos, Ramdy Conrads crea classmates.com, la cual permite mantener y recuperar de com- } \\
\text { pañeros de estudios. }\end{array}$ \\
\hline 1997 & $\begin{array}{l}\text { Continúan los avances y se lanzan al mercado nuevas plataformas, como: } \\
\text { SixDegrees, la cual se sustenta en la Teoría de los Seis Grados } \\
\text { AOL Instant Messenger } \\
\text { Web Sixdegrees.com }\end{array}$ \\
\hline 2000 & La internet \\
\hline 2002 & $\begin{array}{l}\text { Aparecen los primeros sitios web los cuales promocionan las redes de amigos en línea, sitios que } \\
\text { se popularizan rápidamente como el portal Friendster el cual en tres meses llega a tres millo- } \\
\text { nes de usuarios. }\end{array}$ \\
\hline 2003 & Nace la web MySpace, fundada por una empresa dedicada al marketing online \\
\hline 2004 & Surge Facebook \\
\hline 2006 & Aparece Twitter red de microblogging \\
\hline 2011 & $\begin{array}{l}\text { Se fortalecen y posicionan en el mercado Facebook (dominando el mercado), MySpase, Twitter } \\
\text { y Friendster }\end{array}$ \\
\hline
\end{tabular}

Fuente: elaboración propia

Las redes sociales son herramientas estratégicas en el área del marketing de toda empresa, la cual permite anunciarse de manera masiva, ya que estas redes abarcan millones de usuarios a nivel global, lo cual permite una publicidad masiva a bajo costo.

\section{Metodología}

La presente investigación tiene un diseño descriptivo correlacional simple, ya que se buscó encontrar la relación entre el uso del social media marketing y las ventas de las micro-empresas de servicios.

\section{Descripción del área donde se realizó la investigación}

La investigación se realizó en dos vertientes, por una parte, se entrevista- ron a microempresarios que utilizan redes sociales para publicidad y están dirigidos al sector servicios ubicados en la Ciudad de Puebla, por otro lado, se realizaron encuestas a clientes potenciales de empresas que usan redes sociales, para este estudio se eligió a la comunidad universitaria (docentes y alumnos) que estudian o trabajan en Facultad de Administración de la Benemérita Universidad Autónoma de Puebla.

\section{Población y muestra}

En la Ciudad de Puebla, existen muchas microempresas, al igual que en el resto de México, sin embargo, para este estudio era importante que las microempresas se dirigieran específicamente al sector servicios, que su principal mercado meta fuera la co- 
munidad universitaria y que, además, utilizaran social media marketing, es por lo anterior que la muestra se conformó por 15 empresas.

Los criterios de inclusión para la muestra fueron: empresas de menos de 15 trabajadores, pertenecientes al sector servicios (hoteles, restaurantes, agencias de viajes, spa's/clínicas de belleza, servicios médicos), que promocionen sus servicios a través de redes sociales, ubicadas en la Ciudad de Puebla. Así también se investigaron las preferencias de los clientes potenciales de las microempresas de servicios, debido a la ubicación de las empresas seleccionadas, la muestra, está constituida por usuarios de redes sociales, para este estudio se eligieron a estudiantes y docentes de la Facultad de Administración de la BUAP inscritos en el período Primavera 2019. Los criterios de inclusión para la muestra fueron: alumnos y docentes de sexo masculino y femenino, alumnos y docentes de cualquier edad, que cuenten con una red social, que sean clientes potenciales de las microempresas de servicios estudiadas.

Los criterios de exclusión para la muestra fueron: empresas que no acepten participar en el estudio, empresas con mayor número de trabajadores, empresas cuyo mercado potencial sea otro diferente a la comunidad universitaria, empresas de otros sectores como venta de ropa, artículos de tecnología, entre otros, microempresas dedicadas al sector servicios que no utilizan redes sociales como herramienta de marketing. Los criterios de exclusión para la muestra de clientes potenciales fueron: alumnos y docentes que no acepten participar en el estudio, alumnos y docentes que no se encuentren en la facultad al momento de aplicar los cuestionarios.

Según el Anuario Estadístico 20172018 el número total de la población es de 8066. El tipo de muestro con que se trabajará es no probabilístico de conveniencia.

La siguiente fórmula del programa STATS se utilizó para obtener el tamaño de la muestra.

Tamaño del universo $=8066$

Heterogeneidad $=50 \%$

Margen de error $=5 \%$

Nivel de confianza $=95 \%$

Tamaño de la muestra $=367$

\section{Materiales y métodos.}

La metodología utilizada fue una investigación descriptiva correlacional, de acuerdo a esto se decidió elaborar un propio instrumento para la recolección de datos y así facilitar y adecuar su funcionalidad a la investigación.

Hernández Sampieri (2014), señala que la confiabilidad va de la mano con la validez del instrumento, es por eso que se investiga que tipo de validez respaldará las decisiones.

El instrumento utilizado para las entrevistas con dueños y gerentes de las microempresas, consistió en 3 preguntas no estructuradas, para 
permitir que el entrevistado se explayara y poder recabar el mayor número de evidencias posible.

\section{Las preguntas fueron:}

1. Pregunta filtro ¿Utiliza las redes sociales para hacer publicidad de sus servicios?

2. Pregunta 1 ¿Cómo diseñó su estrategia de publicidad en redes sociales?

3. Pregunta 2 ¿Cuál es el impacto que tiene la publicidad en redes sociales para su empresa?

4. Pregunta 3 ¿Cómo mide este impacto?

En el caso de los cuestionarios para la comunidad universitaria (cliente potencial de las microempresas) se desarrolló de la siguiente manera:
Se desarrolló el cuestionario, donde se plantean 7 preguntas con una variedad de opción múltiple en abanico y de estimación, que engloban las posibles respuestas pensando en facilitar la recolección de datos de un modo categórico y sencillo.

\section{Procedimiento}

Para la realización de la investigación se aplicaron encuestas a 362 estudiantes y docentes al azar, los cuales son la muestra que representa a la población total, que formen parte de la Facultad de Administración, dichas encuestas constan de 7 preguntas cerradas y se realizaron durante el mes de marzo de 2019.

Se contó con 14 encuestadores, los cuales se acercarán a los estudiantes y maestros y una vez que la persona entrevistada aceptó participar se

Tabla 2. Instrumento utilizado con la comunidad universitaria.

\begin{tabular}{|c|c|c|c|c|}
\hline Variables & $\begin{array}{l}\text { Definición } \\
\text { operacional }\end{array}$ & Indicadores & Dimensiones & Ítems \\
\hline $\begin{array}{l}\text { Ventas. } \\
\text { Social media } \\
\text { marketing. } \\
\text { Comunidad } \\
\text { universitaria. }\end{array}$ & $\begin{array}{l}\text { Relación del } \\
\text { impacto del } \\
\text { social media } \\
\text { marketing } \\
\text { sobre las ven- } \\
\text { tas de las } \\
\text { micro-empre- } \\
\text { sas que lo uti- } \\
\text { lizan. }\end{array}$ & $\begin{array}{l}\text { Número de com- } \\
\text { pras realizadas a } \\
\text { través de redes } \\
\text { sociales o debido } \\
\text { a la publicidad en } \\
\text { redes sociales. }\end{array}$ & $\begin{array}{l}\text { Social media } \\
\text { marketing. }\end{array}$ & $\begin{array}{l}\text { Pregunta filtro. ¿Utiliza usted } \\
\text { redes sociales? } \\
\text { ¿Ha visto productos o servicios } \\
\text { anunciados en redes sociales? } \\
\text { ¿Cuántas veces ha comprado } \\
\text { productos o servicios anuncia- } \\
\text { dos en redes sociales? } \\
\text { Si su respuesta fue nunca o de } \\
1 \text { a } 3 \text { veces, mencione el motivo } \\
\text { principal por el que no ha com- } \\
\text { prado o ha comprado poco. } \\
\text { ¿Cuál es la red social que más } \\
\text { utiliza? } \\
\text { ¿Qué tipo de anuncios ha } \\
\text { observado por redes sociales? } \\
\text { ¿Cuál es su edad? } \\
\text { ¿Cuál es su género? }\end{array}$ \\
\hline
\end{tabular}

Fuente: elaboración propia 
Propuesta de una herramienta de emprendimiento en el diseña de un madela de negacio, 112-128
Karla Caré Mareno, María Martha del Sacarro Ramano, María del Refugio García les entregó la hoja impresa con las preguntas y una pluma, dentro del cuestionario se escribieron las indicaciones de cómo contestar.

\section{Análisis de datos.}

Según Sampieri (2014) el método de codificación ideal para la mayor parte la encuesta es el de la medición nominal, ya que otorgará a cada respuesta de las preguntas un número, pero el que algún valor sea mayor que otro no necesariamente representa algún tipo de jerarquía, se hace con el propósito de hacer una mejor diferenciación y posterior análisis detallado de los valores obtenidos.

Ya habiendo obtenido todos los datos necesarios se procedió a codificar las respuestas de cada una de las preguntas, a su vez estos resultados se trabajaron en una base de datos, previamente desglosados por pregunta para su posterior análisis.

\section{Resultados y discusión.}

Figura 1. Pregunta filtro ¿Utiliza usted redes sociales?

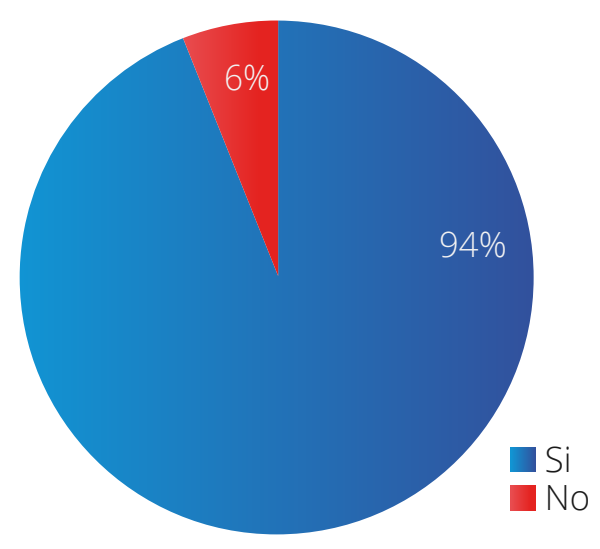

Fuente: elaboración propia.
En este sentido era importante aplicar los cuestionarios sólo a personas que tuvieran redes sociales, se recalca que la gran mayoría de las personas de la población de estudio usan al menos una red social.

Figura 2. Pregunta $1 \mathrm{¿Ha}$ visto productos o servicios anunciados en redes sociales?

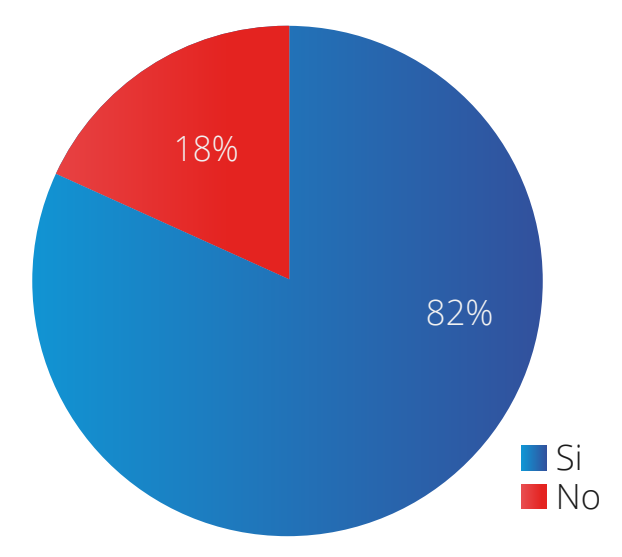

Fuente: elaboración propia.

Existe un alto porcentaje de personas que han visto publicidad en redes sociales, cabe detallar que la mayor parte de esta publicidad, la han encontrado en sitios como Marketplace y similares, es decir publicidad no pagada y a la cual tienen acceso no sólo las empresas, sino también personas físicas que desean revender artículos diversos. Este dato es importante ya que la publicidad que tiene indicadores es la pagada, por tanto, aún no se ve un aprovechamiento generalizado de esta herramienta y existen al momento de la investigación, varias empresas que utilizan publicidad gratuita en redes.

Los datos recabados en estas preguntas (2 a la 5) muestran que la población estudiada aun no compra artículos anunciados en redes sociales, es decir existe gran desconfianza. 
Propuesta de una herramienta de emprendimiento en el diseño de un madelo de negací, II2-128
Karla Laré Morena, María Martha del Sacarro Ramano, María del Refugio García
Figura 3. Pregunta 2 ¿Cuántas veces ha comprado productos o servicios anunciados en redes sociales?

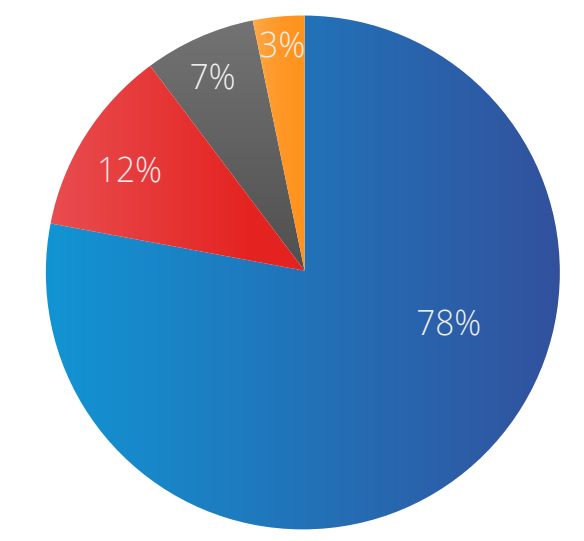

Nunca

De 1 a 3 veces De 6 a 10 veces

De 4 a 5 veces Más de 10 veces

Fuente: elaboración propia.

Figura 4. Pregunta 3 Si su respuesta fue nunca o de 1 a 3 veces, mencione el motivo principal por el que no ha comprado o ha comprado poco

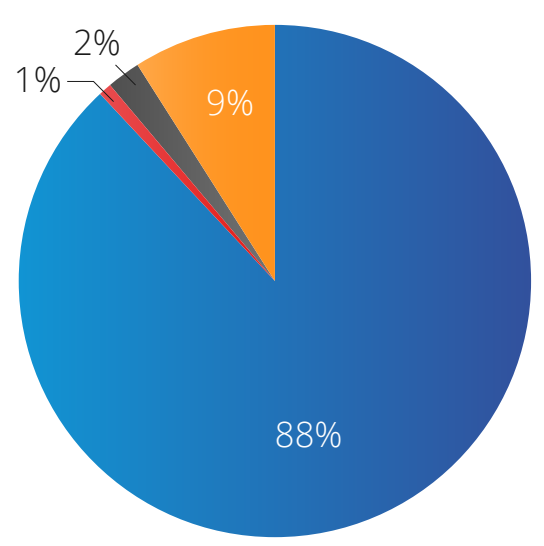

Desconfianza

Falta de claridad en la publicidad

- Precio muy alto

- El producto o servicio no es interesante para mi

Fuente: elaboración propia.
De manera informal se les preguntaba si habían comprado artículos en internet, más del 50 por ciento respondía que sí, pero que lo hacía a través de sitios especializados como Amazon y Mercado Libre.

Figura 5. Pregunta 4 ¿Cuál es la red social que más utiliza?

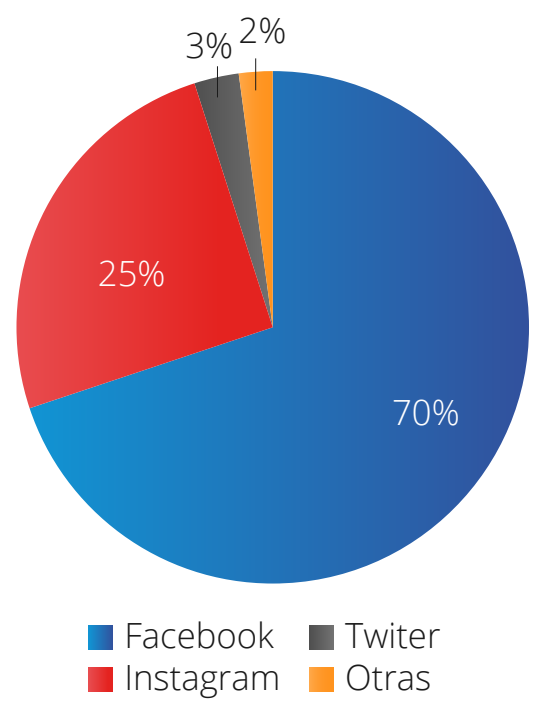

Fuente: elaboración propia.

Como puede observarse la red social más popular entre la población de estudio es Facebook, con un 70 por ciento de las preferencias, sin embargo, Instagram se muestra en un crecimiento rápido en la población más joven.

De igual forma un 43 por ciento de los que compraron alguna vez, mencionaban que los anuncios en redes sociales les ayudaban a atraer su atención hacia los productos, pero no lograban generar en ellos la acción de compra. 
Propuesta de una herramienta de emprendimiento en el diseño de un madelo de negací, II2-128
Karla Laré Morena, María Martha del Sacarro Ramana, María del Refugio García
Figura 6. Pregunta 5 ¿Qué tipo de anuncios ha observado por redes sociales?

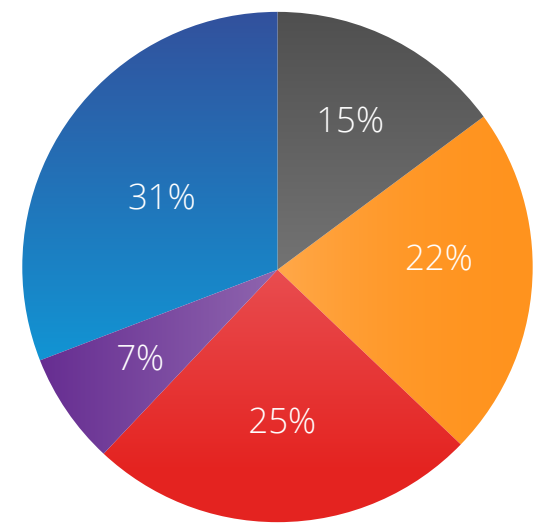

Productos o servicios para la salud - Productos y tecnología

- Viajes, hoteles

- Restaurantes

- Artículos como ropa y zapatos

Fuente: elaboración propia.

Existe un alto porcentaje de artículos como zapatos, ropa y productos de tecnología que aparecen en las redes sociales de la población estudiada, es decir productos, el sector servicios (que es objeto de esta investigación) todavía no explota el social marketing, es decir se encuentra incipiente, siendo los viajes y hoteles, los que más se anuncian de este sector.

Respecto al rango de edad, la mayor parte de la población de estudio está ubicada entre los 19 y 25 años, seguida de los 37 a 50 años, este dato no sorprende, debido a que como se mencionó con anterioridad, la investigación se llevó a cabo en una universidad y la mayor parte de los encuestados fueron alumnos y docentes.
Figura 7. Pregunta 6 ¿Cuál es su edad?

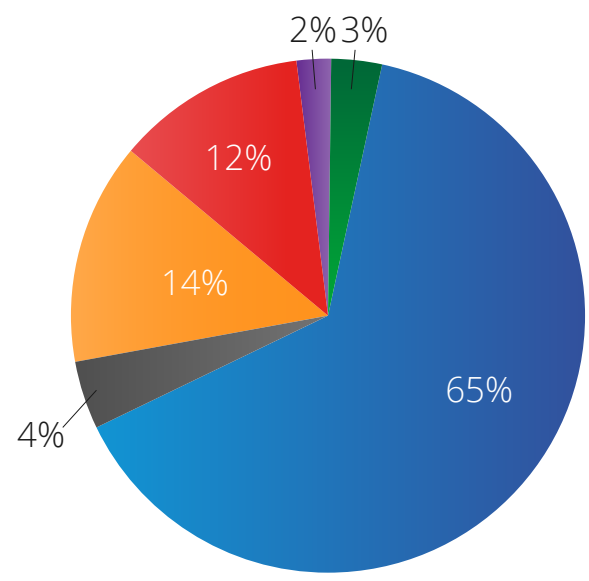

Menos de 18 años

De 19 a 25 años

De 26 a 36 años

De 37 a 50 años

- De 51 a 70 años

— Más de 71 años

Fuente: elaboración propia.

Figura 8. Pregunta 7 ¿Cuál es su género?

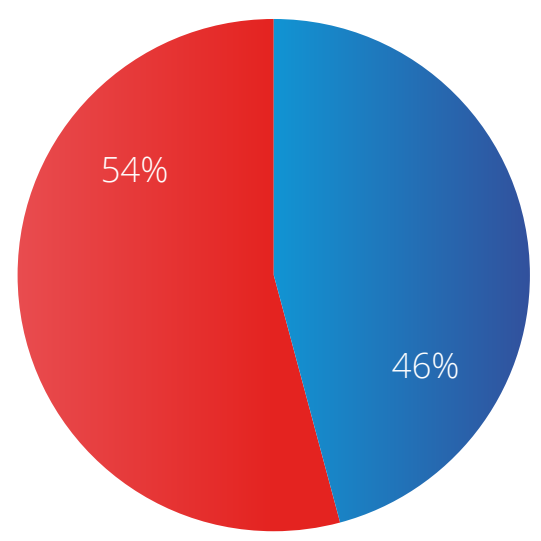

Hombre Mujer

Fuente: elaboración propia.

Debido a la necesidad de conocer el manejo de social media por parte de los microempresarios de servicios que se dirigen a la comunidad universitaria, se buscó entrevistar a 15 
dueños o gerentes de empresas ubicadas en Puebla. Por cuestiones de confidencialidad no se mencionaron los nombres de las empresas, pero la siguiente tabla muestra el número de empleados y sector específico al que se dirigen.

Tabla 3. Relación de empresas que formaron parte del estudio.

\begin{tabular}{ccl} 
Empresa & $\begin{array}{c}\text { Número de } \\
\text { trabajadores }\end{array}$ & Sector específico \\
\hline 1 & 7 & Restaurante \\
2 & 5 & Restaurante \\
3 & 7 & Bar \\
4 & 2 & Estética \\
5 & 15 & Spa \\
6 & 5 & Consultorios médicos \\
7 & 15 & Hotel \\
8 & 5 & Casa de huéspedes \\
9 & 3 & Casa de huéspedes \\
10 & 3 & Agencia de viajes \\
11 & 2 & Dentista \\
12 & 6 & Restaurante \\
13 & 4 & Bar \\
14 & 4 & Restaurante \\
15 & 2 & Cafetería \\
\hline
\end{tabular}

Nota de tabla: elaboración propia.

Pregunta 1 ¿Cómo diseñó su estrategia de publicidad en redes sociales?

El 80 por ciento de las empresas respondió que habían diseñado empíricamente su estrategia de publicidad, es decir, abrieron una página de Facebook o Instagram y sólo suben fotos y promociones pero no les ayudó ningún especialista en social media marketing, la razón generalizada fue porque no lo consideran necesario.

Pregunta 2 ¿Cuál es el impacto que tiene la publicidad en redes sociales para su empresa?
El 87 por ciento de las empresas, respondieron que el impacto en la publicidad en redes sociales es poco, algunos clientes les dicen que han visto sus productos o que los conocen por redes sociales, pero logran traducirlo en ventas.

Pregunta 3 ¿Cómo mide este impacto?

El 80 por ciento de las empresas entrevistadas, no saben cómo medir el impacto del social media marketing, sólo los propietarios que han pagado por la publicidad, reciben estadísticas de cómo y cuánto impacta este en la población, la mayoría solo miden sus resultados por pláticas informales con sus clientes cuando les preguntan ¿cómo supieron de la empresa?

Por los datos recabados es notable que las micro-empresas de servicios no acuden a especialistas para guiarse sobre el social media marketing, el 13 por ciento de los que mencionaron que se apoyaron en un especialista, piensan que la inversión vale por sus beneficios y el otro 7 por ciento aprendió de manera autodidacta a utilizar el social media marketing a través de cursos gratuitos que las propias redes sociales ofrecen cuando se paga por la publicidad.

A pesar de que existen beneficios importantes para las empresas que utilizan el social media marketing, los microempresarios aun no encuentran las estrategias adecuadas para potencializar su impacto.

Como pudo observarse a lo largo de la investigación, la comunidad universitaria como cliente potencial de los 
Propuesta de una herramienta de emprendimiento

en el diseña de un madela de negacia, II2-128
Karla Caré Marena, María Martha del

Socorro Ramana, María del Refugia García microempresarios de servicios, está altamente familiarizada con el uso de redes sociales, sin embargo, no las utilizan como medio para comprar servicios, es incipiente aun el uso del social media marketing en este sector.

Por lo anterior, ante el panorama de que los microempresarios de servicios destinan poco dinero a la contratación de expertos en social media marketing, en conjunto con el hecho de que existen diferentes objetivos de la publicidad en redes sociales, por ejemplo, mientras algunas empresas solo buscan posicionar su marca, otras pretenden tener seguidores en su página y ser recomendados a otros "amigos" y algunas más buscan aumentar las ventas.

Se identificaron las siguientes estrategias del social media marketing que posibilitarán el aumento de las ventas en las microempresas de servicios.

\section{Segmentación adecuada del mer-}

cado. El social media marketing da la oportunidad de segmentar adecuadamente los mercados, cuando una empresa decide hacer publicidad pagada en redes sociales, se le piden datos específicos como: gustos e intereses de los usuarios, edad, rango de movimiento (es decir las calles en las que frecuentemente se encuentra ubicado el usuario), páginas relacionadas a las que se les da me gusta, publicaciones habituales (palabras clave que el usuario publica), búsquedas frecuentes en la red, etc. Es decir, el social media marketing permite identificar de manera casi personalizada al cliente potencial de la empresa, la estrategia que debe seguirse es "aprender a conocer al cliente potencial", si se alimentan adecuadamente todos los datos antes mencionados, es más eficiente el impacto específico de publicidad pagada a través de redes sociales.

\section{Tiempo de recuperación de la in-} versión. Las redes sociales ofrecen diferentes ventajas en materia publicitaria, pero tal como se vio a lo largo del estudio, el impacto no es tan inmediato como los microempresarios esperan, especialmente las empresas que se dirigen al sector servicios, las cuales, por sus características específicas generan mayor desconfianza de compras a través de internet. Entre estas características están el que ofrecen productos intangibles o menos tangibles, el cliente asume un riesgo mayor ya que las garantías son diferentes que en los productos y existe falta de estandarización en la atención y amabilidad del personal, entre otros.

Debido a esto, el tiempo de recuperación de la inversión de social media marketing es de aproximadamente seis meses a dos años, (Marketing Actual.es, 2015) es decir no es una herramienta que traduzca resultados inmediatos en los clientes y así como otros medios de marketing, requiere paciencia y perseverancia por parte de los microempresarios.

3. Diseño adecuado de la publicidad y promoción para redes sociales. Las fotografías de los servicios que se oferten deben 
ser tomadas por profesionales, con cámaras profesionales, en materia de marketing todo comunica y una imagen tomada con celulares o sin manejo de luz y ángulos, poco podrá impactar en el cliente potencial. Las descripciones de los servicios son también fundamentales, en el caso específico de los hoteles, restaurantes y servicios médicos es imposible ofrecer "una prueba" del producto intangible previo a la compra. Lo anterior hace que el consumidor asuma múltiples riesgos como invertir en algo que no sea de su completa satisfacción, es por ello, que todos los elementos que puedan dar certidumbre al comprador, como descripciones que resalten los beneficios obtenidos al usar el servicio, el valor, la satisfacción, las experiencias de otros compradores, serán útiles para aumentar las ventas. Al igual que las palabras, el manejo de colores, la comunicación corporativa, los diseños atractivos de promociones, el tipo y tamaño de letra adecuados a las preferencias del cliente potencial, son elementos que aumentarán la atención del cliente sobre los anuncios publicados.

\section{Otorgue beneficios a los clien- tes que utilizan social media} marketing. Como cualquier otra experiencia, las compras a través de redes sociales, están en una etapa de crecimiento y en algunos usuarios de microempresas de servicios, incluso de introducción; así pues deben ofrecerse incentivos a los clientes que compren a través de estos medios, por ejemplo promociones especiales, descuentos, complementos de servicio, cuando éstos recomienden y utilicen activamente el social media marketing de la microempresa.

\section{Conclusiones}

El social media marketing representa una opción innovadora para posicionar servicios en los consumidores potenciales.

Si bien las estrategias mencionadas en el presente artículo han sido estudiadas en otros similares, se cumplió el objetivo de indagar a usuarios de la comunidad universitaria, y como pudo observarse en los resultados, éstos se muestran poco influenciados ante la publicidad en redes sociales en micro empresas de servicios, sin embargo, es claro que la tendencia irá en aumento y es importante que las microempresas estén preparadas para ello.

Ante los retos que representa el mejorar las ventas de una microempresa de servicios, la investigación constante respecto a las preferencias y el comportamiento del consumidor potencial, seguirá siendo clave para aumentar el market share de las microempresas de servicios y así explotar todos los beneficios que presentan las redes sociales en materia de marketing.

\section{Referencias bibliográficas.}

Adhiambo, Odhiambo, C. (2012) Social Media as a Tool of Marketing and Creating Brand awareness: Case study research Recuperado el 20 de marzo 
de 2019 de: https://www.theseus.fi/ bitstream/handle/10024/44591/Christine.A.Odhiambo.pdf?sequence=1

Abdul, S. R., \& Nor, A. B. (2016). Factors That Influence The Usage of Social Media In Marketing. Journal of Research in Business and Management. Vol.4 No.2. Page 01-07.

Álvares Rengifo, G. R., \& Bendezú Sánchez, R. M. (2011). Tendencias de marketing digital 2018: Recuperado el 19 de abril de 2019, de https://www.kanlli. com/estrategia-marketing-digital/tendencias-de-marketing-digital-2018/

Benemérita Universidad Autonóma de Puebla. (2017). Anuario Estadístico Institucional. Puebla: BUAP: Recuperado el 19 de abril de 2019 https:// www.pdi.buap.mx/sites/default/files/ pdf/1er_informe_2018/anuario_estadistico.pdf

Díaz, V. (30 de 09 de 2019). Generación de leads: Todo lo que debes saber. Recuperado el 19 de abril de 2019 de https://neoattack.com/generacion-de-leads/

Hernandez, Sampieri et al. (2014) Metodología de la Investigación. México: Mc Graw Hill.

Marketing Actual.es. (29 de 04 de 2015). Obtenido de 12 objetivos de Social Media Marketing para las marcas: http://marketingactual.es/social-media/social-media/12-objetivos-de-social-media-marketing-para-las-marcas

Gutiérrez-Leefmans, Manuela, \& Patrick Holland, Christopher. (2019). SME Platforms as Business Models: A User-Centric Activity-System Approach. Management (Universidad del Valle), 35(64), 52-77. https://dx.doi. org/10.25100/cdea.v35i64.7248

Hudson, S., \& Thal, K. (2013). The impact of social media on the consumer deci- sion process: Implications for tourism marketing. Journal of Travel \& Tourism Marketing, 30(1-2), 156-160.

Kumalasari Alloq, D (2018). The role of social media as marketing tools for small and medium enterprises performance. Recuperado el 11 de abril de 2019 de: http://administrasibisnis.studentjournal.ub.ac.id/index.php/jab/article/ view/2688/3082

Ladkin, A., \& Buhalis, D. (2016). Online and social media recruitment: Hospitality employer and prospective employee considerations. International Journal of Contemporary Hospitality Management, 28(2), 327-345.

Méndez, F y Mendoza, D. (14 de enero de 2018). UNAM global. Recuperado el 19 de abril de 2019, de http://www. unamglobal.unam.mx/?p=31030

Mehta. (2017). Integrated Marketing Communications at Nike. Slideshare. net. Recuperado el 5 de septiembre de 2018, de https://www.slideshare. net/KaranMehta28/integrated-marketing-communications-at-nike

Miranda Zavala, Ana María, \& Cruz Estrada, Isaac. (2016). Redes sociales: herramienta de marketing internacional en el sector hotelero. El periplo sustentable, (31), 00012. Recuperado en 01 de octubre de 2019, de http://www.scielo.org.mx/ scielo.php?script=sci_arttext\&pi$d=S 1870-90362016000200012 \&$ In$\mathrm{g}=\mathrm{es} \& \operatorname{lng}=\mathrm{es}$.

Miles, C. (2013). Persuasion, marketing communication, and the metaphor of magic. European Journal of Marketing, 47(11/12), 2002-2019.

Moreno Cortés, K., Maldonado Reséndiz, J., \& Morales Espíndola, G. (2017). Factores que influyen en la selección 
de restaurantes (consumidores), del centro histórico de Puebla. Revista GEON (Gestión, Organizaciones Y Negocios), 4(2), 71-80. https://doi. org/10.22579/23463910.24

NeoAttack. (30 de 09 de 2019). Obtenido de SOCIAL MEDIA MARKETING: https://neoattack.com/neowiki/social-media-marketing/

Peña, K., Pérez, M., \& Rondón, E. (2010). Redes sociales en Internet: reflexiones sobre sus posibilidades para el aprendizaje cooperativo y colabirativo. Revista de Teoría y Didáctica de las Ciencias SOciales, 173-205.
Ruiz de Querol, R. y. (2007). La Sociedad de la informatica. Barcelona: UOC.

Valencia, J. R. (1999). Facultad de Administración de UAP. Gaceta Histórica de la Benemérita Universidad Autónoma de Puebla(10).

Velazquez, K. (11 de 12 de 2015). Marketing 4ecommerce.mx. Obtenido de https://marketing4ecommerce. $\mathrm{mx} /$ que-es-social-media-marketing-definicion-y-tendencias/

Zorraquino, D. (30 de 09 de 2019). Diccionario Zorraquino. Obtenido de https://www.zorraquino.com/diccionario/marketing-digital/que-es-social-media-marketing.html 